\title{
Case Report: Endoscopic Management of Sleeve Gastrectomy Fistula
}

\author{
Roșu Alexandra Floriana1, Ferreira Carlos Noronha², Ribeiro Luis Carrilho², Velosa José2, Zlatian Ovidiu \\ Mircea $^{3}$
}

1 Gastroenterology Department, County Clinical Emergency Hospital, Craiova, Romania

${ }^{2}$ Gastroenterology and Hepatology Department, Universitary Hospital Santa Maria, Lisbon, Portugal

${ }^{3}$ Microbiology department, University of Medicine and Pharmacy of Craiova, Romania

Background. Morbid obesity is an important cause of morbidity and mortality. Bariatric surgery is the best option to manage obesity. Vertical gastrectomy is safe and effective but sometimes complicate with hemorrhage, fistulas and stenosis. Fistulas can be solved by conventional surgery or interventional endoscopy.

Case presentation. We describe a morbidly obese patient with vertical gastrectomy who developed complications after surgery. Immediately after surgery the patient developed sepsis. Upper gastrointestinal endoscopy excluded fistula. One month later a peri-gastric abscess developed due to a fistula orifice in the distal esophagus, treated with argon plasma and two double pigtail plastic stents placed endoscopically to drain the abscess. The stents were removed two weeks later and was placed a covered metallic stent in the distal esophagus. Six weeks later the metallic stent was removed and the orifice closed. Four months later the patient developed sepsis. Computer tomography revealed a subdiaphragmatic abscess and endoscopy revealed a $2 \mathrm{~mm}$ fistula orifice at the previous site, treated with argon plasma and two troughthe-scope clips that closed it. There were no further incidents after two years of follow-up.

Conclusions. Early diagnosis and endoscopic approach can resolve these complications without the morbidity and increased mortality risk of surgical re-interventions.

Keywords: sleeve gastrectomy, bariatric surgery, endoscopic treatment

Received 07 December 2015 / Accepted 04 November 2016

\section{Introduction}

Obesity is a serious health problem associated with genetic, environmental and hormonal causes that has doubled in prevalence since 1980 and affects 1.4 billion adults [1]. Obesity increase risk of diabetes, hypertension, hyperlipidemia, sleep apnea, osteoarthritis, and other degenerative diseases [2].

Dietary therapy is ineffective in morbid obesity as the weight loss is about $5-10 \%$ [3]. Consequently, bariatric surgery has become popular, due to its effectiveness in terms of weight loss and reduction of associated comorbidities [4].

The most used procedures are Roux-en-Y gastric bypass (RYGB), adjustable gastric banding (AGB) and vertical gastrectomy $(\mathrm{VG})$ also known as sleeve gastrectomy (SG) [5]. Biliopancreatic diversion / duodenal switch is best for extremely obese individuals [6]. All procedures can be performed laparoscopically with a low rate of complications [7].

AGB and VG are considered restrictive procedures, VG has metabolic effects comparable with RYGB $[4,8]$. VG involves removal of a $75 \%$ of stomach resulting a sleeve or tube like structure [9].

VG is safe and effective with less operating time (76.6 $+/$ - 28.0 minutes versus $106.2+/-33.2$ minutes) and short-

* Correspondence to: Alexandra Floriana Rosu

E-mail: alexandrarosu13@gmail.com er hospitalization $(2.8+/-.8$ days versus $3.4+/-4.4$ days $)$ compared with RYGB [4, 10]. VG complications include hemorrhage, anastomotic leaks and stenosis, at lower rates in SG (2.9\% versus $7.1 \%)$ compared to RYGB [4].

One frequent complication of bariatric surgery is digestive fistula, often at the cardia. Post-gastrectomy fistulas associate with increased mortality rates, mainly due to development of abdominal abscesses and sepsis. Vertical gastrectomy is associated with an incidence of fistulas of $0.5-2.9 \%[8]$.

\section{Case report}

We describe the case of a morbidly obese patient who underwent vertical gastrectomy. The patient developed sepsis in the immediate post-operative period. Upper gastrointestinal endoscopy didn't found a fistula orifice and a nasogastric tube was placed. The patient improved significantly on broad spectrum antibiotics and was discharged after 10 days.

After one month the patient returned with fever. Abdominal computer tomography showed a fluid collection in the perisplenic and left subphrenic area with gas. The extravasation contrast suggested a fistula near the esophagogastric junction (Figure 1).

Endoscopy revealed a $8 \mathrm{~mm}$ fistula orifice in distal esophagus communicating with a large peri-gastric collection. Pus was aspirated from the abscess and two plastic double pigtail $10 \mathrm{~F}$ stents were placed to drain the collec- 


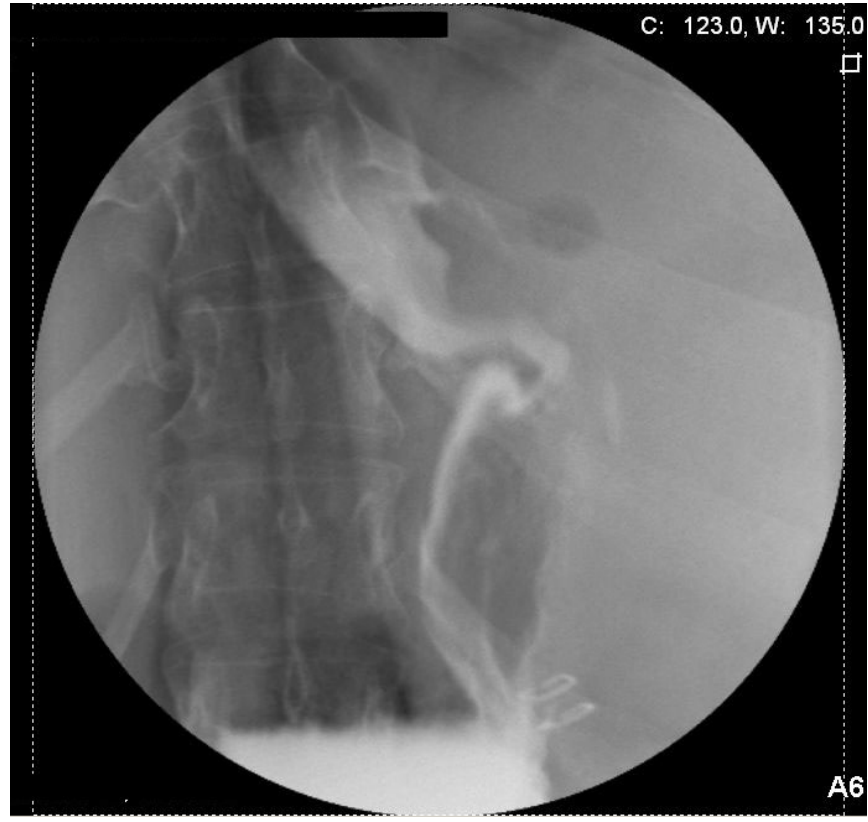

Fig. 1. Endoscopical image of the fistula orifice

tion into esophageal lumen (Figure 2). After antibiotic treatment the acute phase markers normalized in 10 days.

Two weeks later, the plastic stents were removed endoscopically, fistula orifice was closed with argon plasma and was placed a partially covered metallic stent "Ultraflex" 23/28x $120 \mathrm{~mm}$. Gastrografin imaging confirmed exclusion of the fistula orifice (Figure 3). The patient was discharged after a week with indications of diet without fibers. The stent was removed 6 weeks later and the fistula orifice had healed (Figure 4).

Four months later the patient returned with fever and computer tomography revealed a subdiaphragmatic abscess. Gastroduodenal endoscopy revealed a fistula orifice of $2 \mathrm{~mm}$ at the previous site. This was treated with argon

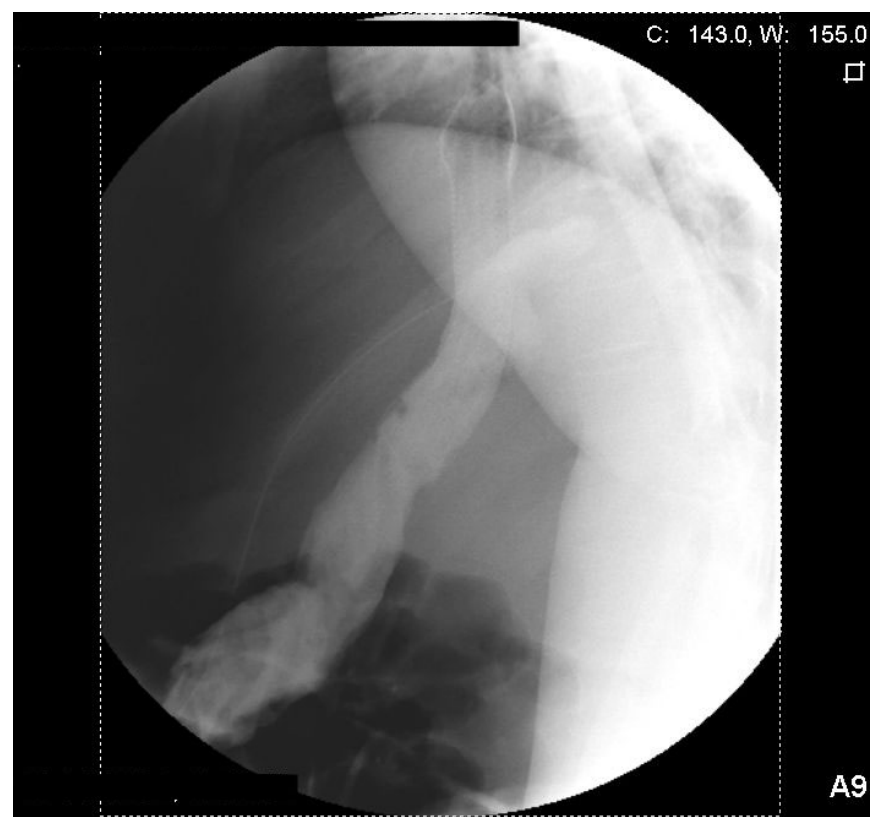

Fig. 3. Gastrografin contrast study confirming effective exclusion of the fistula orifice by the partially covered metallic stent

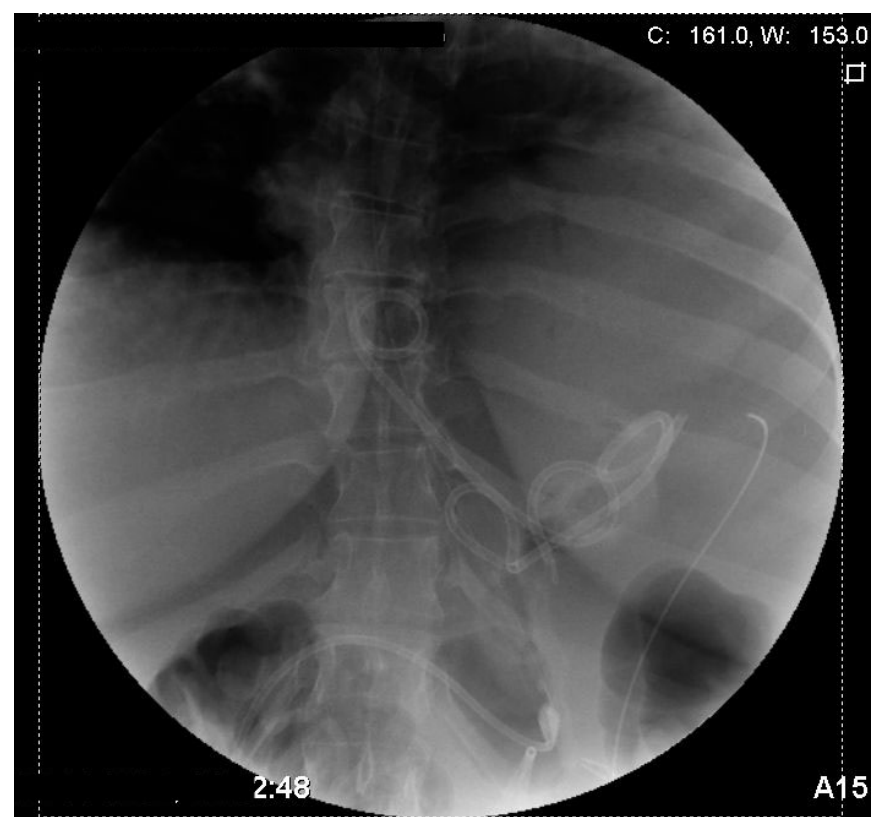

Fig. 2. Double pigtail stents placed endoscopically to drain the perigastric abcess

plasma coagulation and were placed two trough-the-scope clips (TTSC), closing the fistula (Figure 5, Figure 6). The patient was discharged after 10 days of antibiotic therapy. There have been no further incidents after two years of follow-up.

\section{Discussion}

Laparoscopic vertical gastrectomy is popular due to excellent results in terms of weight loss and effective control of hypertension, hyperlipidemia and diabetes mellitus. In one study, VG decreased excess body weight by $49.1 \%$ after 6 months and other comorbidities: hypertension resolved in 38\%, hyperlipidemia resolved in $19 \%$ and diabetes in 46\% [11]. Nevertheless, VG is associated with a

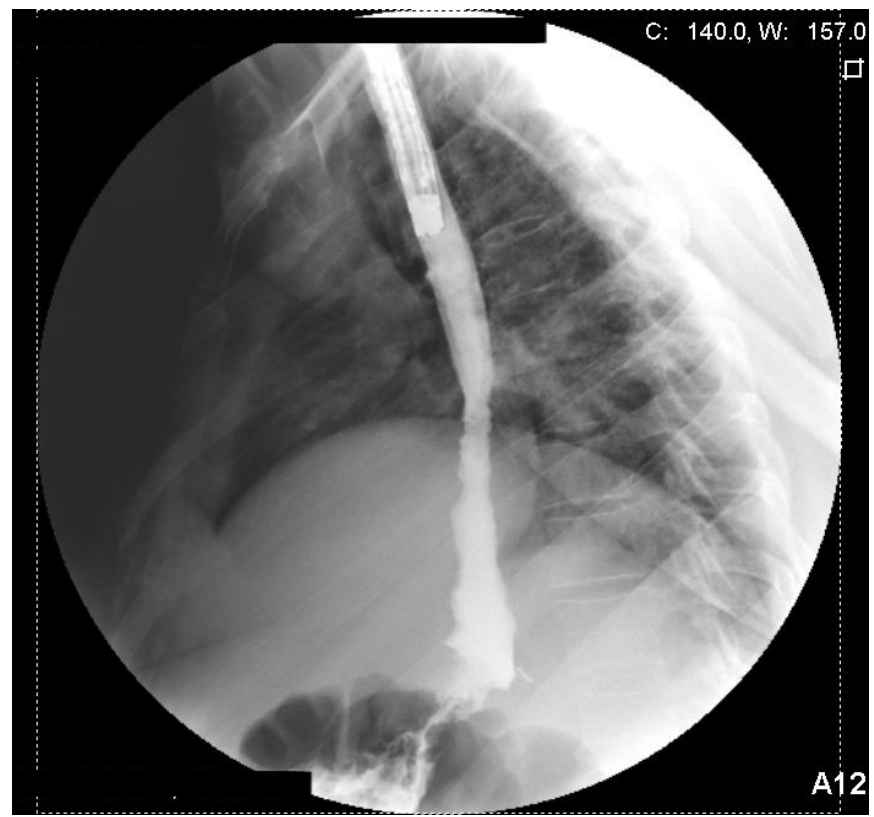

Fig. 4. Gastrografin contrast study after removal of the metallic stent, confirming the closure of the fistula orifice 


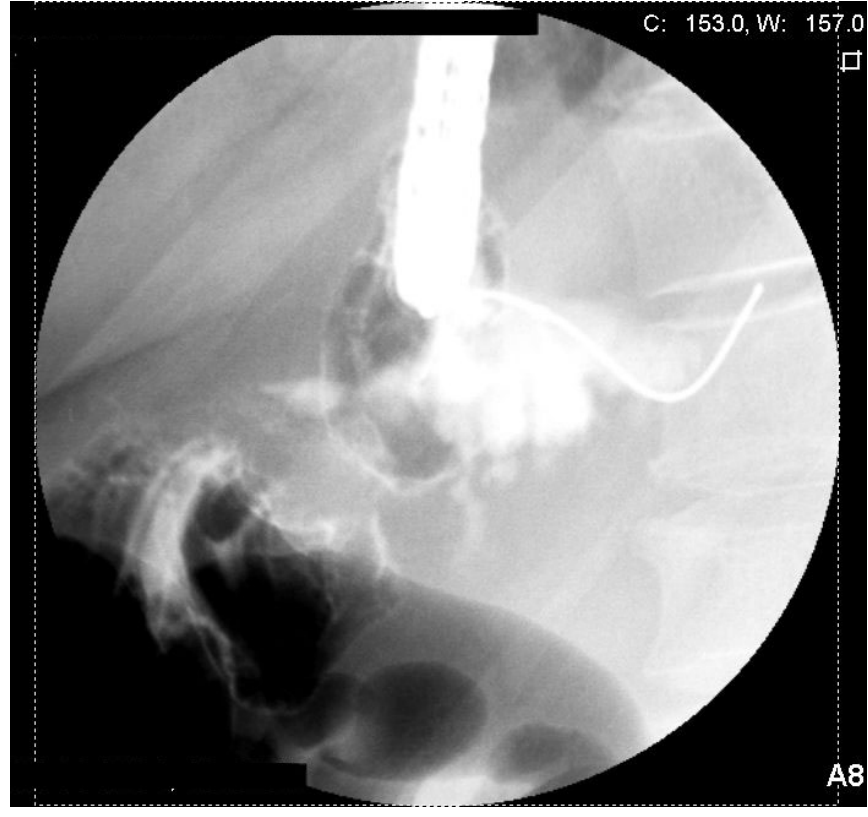

Fig. 5. Gastrografin contrast study confirming effective closure of the recurrent fistula orifice by the TTS clips

$9 \%$ risk of complications with a mortality rate of $0.6 \%$ in USA $[8,12]$.

The most frequent complications of VG are fistulas at esophagogastric junction [9], most commonly at the left cardia, the uppermost region where gastric stapling occurs [13-15].

Digestive fistulas can result in prolonged hospitalization, severe repercussion on general wellbeing and emotional stress $[9,15]$.

Factors that favor the fistulas are local - poor technique, drains, and systemic - nutritional state and corticoid treatments. Other risk factors include patient's age, the level of plasma proteins, systemic sepsis and neoplasia [16].

The pathogenesis of the gastric fistulae involves iatrogenic gastric injury or disruption of the gastric suture lines. Some researchers suggest that the underlying mechanism is poor vascularization due to compromised blood supply which prevents complete healing of the suture [17].

Consequences of the fistula are fluid losses, metabolic disorders, loss of enzymes and electrolytes, inflammation and sepsis $[9,17,18]$. Management of these complications requires good team work between surgeons, gastroenterologists, intensive care specialists and interventional radiologists.

Conventional management of fistulas involves surgical closure of fistula. The suturing is often ineffective because of inflammation. Surgical possibilities are limited for fistulas close to the esophagogastric junction. Emergency surgery is indicated for hemodynamically unstable or septic patients and patients with complicated leaks where drainage under endoscopic or radiological control is not feasible $[15,19]$. A radical intervention is the conversion of $\mathrm{VG}$ to RYGP, which has a 33\% rate of fistula persistence [20].

After SG is recommended endoscopical assessment [21] with identification of fistula orifice and trajectory, assess-

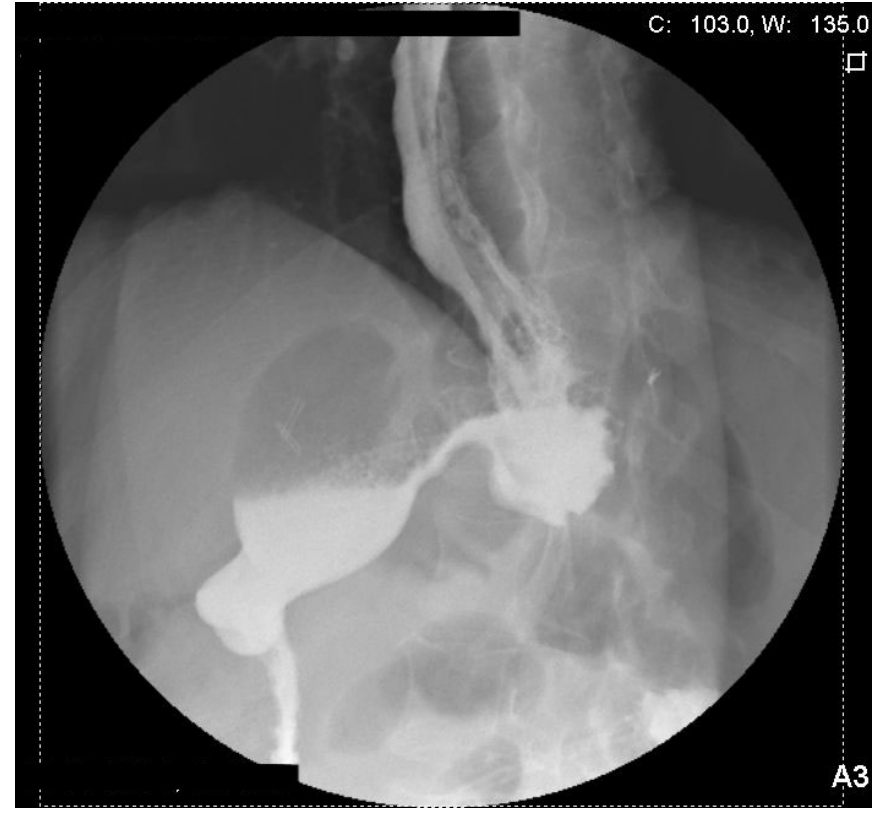

Fig. 6. Gastrografin contrast study at 3 weeks follow-up, confirming the closure of fistula orifice

ment of topographic relations and imaging (fistulography) [21]. Interventional endoscopy is less invasive, isn't affected by BMI and doesn't induce local inflammation that interfere with healing [20].

Endoscopic approach of fistulas post-VG involves localization of the fistula site, debridement and drainage of abscesses, diversion of the fistula orifice and closure of persistent leaks $[17,21]$.

In some centers, post-SG fistulas are managed by identification of the fistula orifice and tract, followed by sealing the inner orifice with fibrin glue [22].

Another option is to place a covered metallic stent which has the advantage of improving and simplifying management, since stents immediately exclude the leak and prevent additional contamination of the peritoneal cavity [22].

Oral nutrition can be started after stent placement, avoiding parenteral nutrition associated with increased risk of infections $[20,22]$.

Fistulas can be managed conservatively with external drainage, gastric decompression with nasogastric catheter, parenteral nutrition, antisecretory drugs and systemic antibiotics [20].

An efficient option seems combination of different procedures like glue and stent placement [21], particularly in cases of pleural or bronchial communication. The re-intervention for gastric fistulas have a cure rate of about $73 \%$, but exists a risk of unfavorable evolution which requires prolonged hospitalization and antibiotic treatment [18].

A study by Ghotb et al., reported 31 cases of postsurgical gastrointestinal leaks and fistulas occurring after different types of surgeries like VG (52\%), RouxN-Y gastric bypass (20\%), duodenal switch (12\%) and VG combined with duodenal switch (16\%). Multimo- 
dality endoscopic therapy included injection [fibrin glue or cyanoacrylate], endoprosthesis (fully covered plastic or metal stents), endoclips and T-tag placement were used. Healing rate doesn't depend on type of surgery or fistula size at presentation and no complications were reported [23].

\section{Conclusions}

This case illustrates the need for a high index of suspicion of gastric fistulas after SG. Early diagnosis and endoscopic approach in management of these complications is essential to prevent relapses and shorten the hospitalization period.

\section{Conflict of interest}

None to declare.

\section{References}

1. World Health Organisation. Obesity and overweight World Health Organisation; 2011. Available from: http://www.who.int/mediacentre/ factsheets/fs311/en/.

2. Proven strategies for combating excess weight. Obesity-related health problems and cardiovascular risks can be reduced with weight loss. The Johns Hopkins medical letter health after 50, 2014;26(9):6.

3. Tokunaga K, Furubayashi T. [Dietary therapy for obesity]. Nihon rinsho Japanese journal of clinical medicine, 2013;71(2):315-9.

4. Boza C, Gamboa C, Salinas J, Achurra P, Vega A, Perez G. Laparoscopic Roux-en-Y gastric bypass versus laparoscopic sleeve gastrectomy: a case-control study and 3 years of follow-up. Surgery for obesity and related diseases, 2012;8(3):243-9.

5. Espinet-Coll E, Nebreda-Duran J, Gomez-Valero JA, Munoz-Navas M, Pujol-Gebelli J, Vila-Lolo C, et al. Current endoscopic techniques in the treatment of obesity. Rev Esp Enferm Dig, 2012;104(2):72-87.

6. Gersin KS, Rothstein RI, Rosenthal RJ, Stefanidis D, Deal SE, Kuwada TS, et al. Open-label, sham-controlled trial of an endoscopic duodenojejunal bypass liner for preoperative weight loss in bariatric surgery candidates. Gastrointestinal endoscopy, 2010;71(6):976-82.

7. Reoch J, Mottillo S, Shimony A, Filion KB, Christou NV, Joseph L, et al. Safety of laparoscopic vs open bariatric surgery: a systematic review and meta-analysis. Arch surg, 2011;146(11):1314-22.

8. Zhang C, Yuan Y, Qiu C, Zhang W. A Meta-analysis of 2-Year Effect After Surgery: Laparoscopic Roux-en-Y Gastric Bypass Versus Laparoscopic Sleeve Gastrectomy for Morbid Obesity and Diabetes Mellitus. Obesity surgery, 2014.

9. Mittermair R, Sucher R, Perathoner A. Results and complications after laparoscopic sleeve gastrectomy. Surg Today 2013. doi: 10.1007/ s00595-013-0688-0.

10. Gagner M, Deitel M, Kalberer TL, Erickson AL, Crosby RD. The Second International Consensus Summit for Sleeve Gastrectomy, March 19-21, 2009. Surgery for obesity and related diseases, 2009;5(4):476-85.

11. Nath A, Leblanc KA, Hausmann MG, Kleinpeter K, Allain BW, Romero R. Laparoscopic sleeve gastrectomy: our first 100 patients. Journal of the Society of Laparoendoscopic Surgeons, 2010;14(4):502-8.

12. Spaniolas K, Trus TL, Adrales GL, Quigley MT, Pories WJ, Laycock WS. Early morbidity and mortality of laparoscopic sleeve gastrectomy and gastric bypass in the elderly: a NSQIP analysis. Surgery for obesity and related diseases, 2014, 10:14-19.

13. Herron D, Roohipour R. Complications of Roux-en-Y gastric bypass and sleeve gastrectomy. Abdom imaging, 2012;37(5):712-8.

14. Rebibo L, Dhahri A, Verhaeghe P, Regimbeau JM. Early gastric fistula after laparoscopic sleeve gastrectomy: surgical management. Journal of visceral surgery, 2012;149(5):e319-24.

15. Sarkhosh K, Birch DW, Sharma A, Karmali S. Complications associated with laparoscopic sleeve gastrectomy for morbid obesity: a surgeon's guide. Can J Surg, 2013;56(5):347-52.

16. Campos AC, Meguid MM, Coelho JC. Factors influencing outcome in patients with gastrointestinal fistula. The Surgical clinics of North America, 1996;76(5):1191-8.

17. Falconi M, Pederzoli P. The relevance of gastrointestinal fistulae in clinical practice: a review. Gut, 2001;49 Suppl 4:iv2-10.

18. Moszkowicz D, Arienzo R, Khettab I, Rahmi G, Zinzindohoue F, Berger A, et al. Sleeve gastrectomy severe complications: is it always a reasonable surgical option? Obesity surgery, 2013;23(5):676-86.

19. Ben Yaacov A, Sadot E, Ben David M, Wasserberg N, Keidar A. Laparoscopic total gastrectomy with Roux-y esophagojejunostomy for chronic gastric fistula after laparoscopic sleeve gastrectomy. Obesity surgery 2014;24(3):425-9.

20. van de Vrande S, Himpens J, El Mourad H, Debaerdemaeker R, Leman $\mathrm{G}$. Management of chronic proximal fistulas after sleeve gastrectomy by laparoscopic Roux-limb placement. Surgery for obesity and related diseases, 2013;9(6):856-61.

21. Bege T, Emungania O, Vitton V, Ah-Soune P, Nocca D, Noel P, et al. An endoscopic strategy for management of anastomotic complications from bariatric surgery: a prospective study. Gastrointestinal endoscopy, 2011;73(2):238-44.

22. Kouklakis G, Zezos P, Liratzopoulos N, Gatopoulou A, Oikonomou A, Pitiakoudis M, et al. Endoscopic treatment of a gastrocutaneous fistula using the over-the-scope-clip system: a case report. Diagnostic and therapeutic endoscopy, 2011;2011:384143.

23. Ghotb A, Shah JN, Binmoeller KF, Weilert F, Bhat YM. Tu1394 Efficacy of Endoscopic Treatment of Symptomatic Upper Gastrointestinal Leaks and Fistulas Post-Bariatric Surgery: Report of 25 Cases. Gastrointestinal endoscopy, 2011;73(4):AB395. 